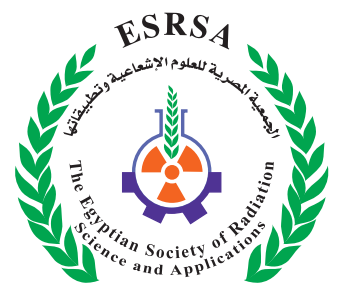

J. Nucl. Tech. Appl. Sci., Vol. 8, PP. 97 : 111 (2020)

\title{
Artisanal Gold Mining Activity in Northcentral Nigeria and Its Implications: Radiological Approach
}

Gomina, M. ${ }^{1,2}$; Kolo, M. T. ${ }^{1}$; Awojoyobge, O. B. ${ }^{1}$ and Olarinoye, O. ${ }^{1}$

Received: 08/05/2020

Accepted: 04/08/2020

DOI: $10.21608 /$ jntas.2020.29717.1021

E.mail:matthewkolo@futminna.edu.ng

\section{ABSTRACT}

Artisanal gold mining is one of the anthropogenic activities identified by the United Nations Scientific Committee on the Effects of Atomic Radiation as potential source of exposure to naturally occurring radionuclides. 40 surface soil samples randomly collected from Gababiyu artisanal gold mining site, in Minna Metropolis (Nigeria), were assessed for their natural radioactivity using gamma spectrometric technique which employs a $\mathrm{NaI}(\mathrm{Tl})$ gamma-ray detector. The specific activities of ${ }^{226} \mathrm{Ra}$, ${ }^{232} \mathrm{Th}$ and ${ }^{40} \mathrm{~K}$ in the soil varied from $10.272 .88 \pm \mathrm{Bq} / \mathrm{kg}$ to $152.60 \pm 3.80$ $\mathrm{Bq} / \mathrm{kg}, 32.67 \pm 1.93 \mathrm{~Bq} / \mathrm{kg}$ to $185.90 \pm 6.06 \mathrm{~Bq} / \mathrm{kg}$ and $35.18 \pm 1.45 \mathrm{~Bq} / \mathrm{kg}$ to $947.50 \pm 7.51 \mathrm{~Bq} / \mathrm{kg}$ respectively, with mean values of $65.06 \pm 4.20 \mathrm{~Bq} / \mathrm{kg}$, $87.63 \pm 2.89 \mathrm{~Bq} / \mathrm{kg}$ and $267.94 \pm 4.29 \mathrm{~Bq} / \mathrm{kg}$ in sequence. Although ${ }^{226} \mathrm{Ra}$ and ${ }^{232} \mathrm{Th}$ recorded activity values slightly above the world average, they are still within the safety range prescribed by UNSCEAR. The computed average absorbed dose rate at $1 \mathrm{~m}$ above the ground was $94.16 \mathrm{nGy} / \mathrm{h}$ with a corresponding mean annual dose equivalent of $0.12 \mathrm{mSv} / \mathrm{y}$. Furthermore, calculated average excess lifetime cancer risk was found to be $0.40 \times 10^{-3}$, which is slightly above the UNSCEAR safety limit. Pearson correlation statistics identified ${ }^{226} \mathrm{Ra}$ and ${ }^{232} \mathrm{Th}$ as principal radionuclides responsible for the computed radiation risk variables. Although the results of this investigation does not show any immediate radiological risk, continuous monitoring of the gold mining site is encouraged in order to keep the radiation effects as low as reasonably achievable.

\section{Artisanal gold mining, Excess lifetime cancer risk, NORM, Northcentral Nigeria, $\mathrm{NaI}(\mathrm{Tl})$ gamma detector. \\ KEYWORDS}




\section{INTRODUCTION}

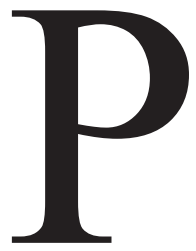

eople's life and development requires natural resources which are randomly distributed in the earth crust (Candeias et al., 2018). Mining and mineral extraction occurs wherever natural resources (metallic, non-metallic minerals and fossils) are located and at economically viable amount (Ako et al., 2014). When the activities are properly done and in compliance with the regulations established by the government and other regulatory agencies, radioactive mineral extractions can be of great benefit to the population. Aside jobs creation, mining and mineral extraction activities are viable sources of economic development and foreign exchange earnings for any nation. One of the most precious and economically viable natural resources that has attracted continuous attention from miners and investors is the gold. On a large scale, gold-mining can be a promising means of economic development and foreign exchange earnings for any nation. Artisanal (small-scale) mining is any activity that encompasses small, medium, informal, legal and illegal mining that involves the use of rudimentary methods and processes to extract mineral resources (Sabo et al., 2018).

Mining and mineral extraction processes have been found over time to incite depletion of the environment such as land degradation, de-vegetation, air and water pollutions and loss of aquatic organisms (Ako et al., 2014). Additionally, these processes have been known to propagate and concentrate natural radioisotopes within the environment to such levels that may become detrimental to human health (Kolo et al., 2015, Kolo et al., 2016). Environmental impact of artisanal gold mining in developing countries particularly in West Africa has been well documented (De Lacerda \& Salomons, 2012; Hilson, 2002; Hollaway, 1993; Meech et al., 1998; Mireku-Gyimah \& Suglo, 1993).

Nigeria is a gold-rich country. Gold deposits are found in most parts of northern Nigeria with an aver- age deposit of 21.40 tons (Okore, 2018). Gold Mining in Nigeria resumed in the 1960s, but could not be developed as rapidly as expected due to the Civil war between 1967 and 1970. In the 1980s, however, there was a rebirth of this precious metal mining as a result of the efforts of the Nigerian Mining Corporation. Yet, poor attention from the government regarding this mineral resource exploitation, unemployment and extreme poverty has driven large number of socially and economically marginalized communities to adopt local mining activity as their main occupation (Pure Earth., 2008). Several gold-rich rural areas in Nigeria have been dominated by unskilled artisanal miners who are underequipped and have little appreciation for the environment (Sabo et al., 2018). Most of these artisanal miners are totally ignorant of, or uninformed about the implications of mining activities on human health and the environment. Therefore, management of radionuclide enriched mine wastes has become a critical challenge in mining sites. UNSCEAR (2000) identified mining and mineral exploitation processes as one of the potential sources of exposure to naturally occurring radioactive materials (NORM) (Faanu et al., 2016). Exposure to ionizing radiation emitted by NORM poses radiological risk to humans and the environment (Alharbi, 2016). The probability and nature of corresponding effects (somatic or genetic) induced in any human population depend on the radiation dose received (NYSDH, 2007).

Gababiyu in North-Central Nigeria has been known for artisanal gold mining activities for decades, with poor or no attention paid to radiation safety guidelines by the local miners. Gold mine wastes are dumped haphazardly all-round the mine by the local miners and thus become avenues for radiological contamination of the soil environment. The locals have constant and continuous access to the mining site as a result of their agricultural activities, which leads to their constant exposure to radiation. Consumption of food grown on this contaminated soils is an additional pathway for radiation 
exposure. Furthermore, soils from Gababiyu mining site have been used by the locals on regular basis, either for landfills or as aggregates of building materials, thereby enhancing their chances of constant radiation exposure.

Almost nothing is known about basic data and information on the radiological implications and associated radiological risk due to artisanal gold mining activities in Gababiyu mining site. We consider that research trials in this area will be useful to the industrial sector of Nigeria government. This study is therefore, aimed at assessing the radiological implications of artisanal gold mining activities in Gababiyu artisanal gold mine site. It is considered a pilot study, whose results will assist the Nigerian government and regulatory authorities in providing comprehensive radiological safety management protocols to keep radiation effects due to artisanal mining activities as low as reasonably achievable, considering economic and social factors.

\section{MATERIALS AND METHOD}

\section{Sample Site}

Gababiyu artisanal goldmine is located between longitude $6^{\circ} 20^{\prime} 00^{\prime \prime}$ to $6^{\circ} 37^{\prime} 30^{\prime \prime} \mathrm{E}$ and latitude $9^{\circ}$ $32^{\prime} 30^{\prime \prime}$ to $9^{\circ} 42^{\prime} 30^{\prime \prime} \mathrm{N}$, in Chanchaga local government area, of Minna, Niger State, in North-Central Nigeria. It is characterized by two distinct seasons namely, rainy (from April/May through October) and dry (December - March), with the two seasons often separated by somewhat transitional periods in April and November. The mean annual rainfall is about $1284 \mathrm{~mm}$, with temperature range of $27^{\circ} \mathrm{C}$ to $33^{\circ} \mathrm{C}$. The site (Figure 1), which is characteristically grass-dominated, forming a mixture of the southern and northern Guinea Savanna ecological biomes, falls within the temperate humid zone that coincides with the tropical hinterland and Guinea Savanna zone of Nigeria. The site which is geologically surrounded by rugged terrain of granitic rocks, is part of Minna Sheet 164 of the Basement Complex Terrain of Nigeria (Ahmed et al., 2019).

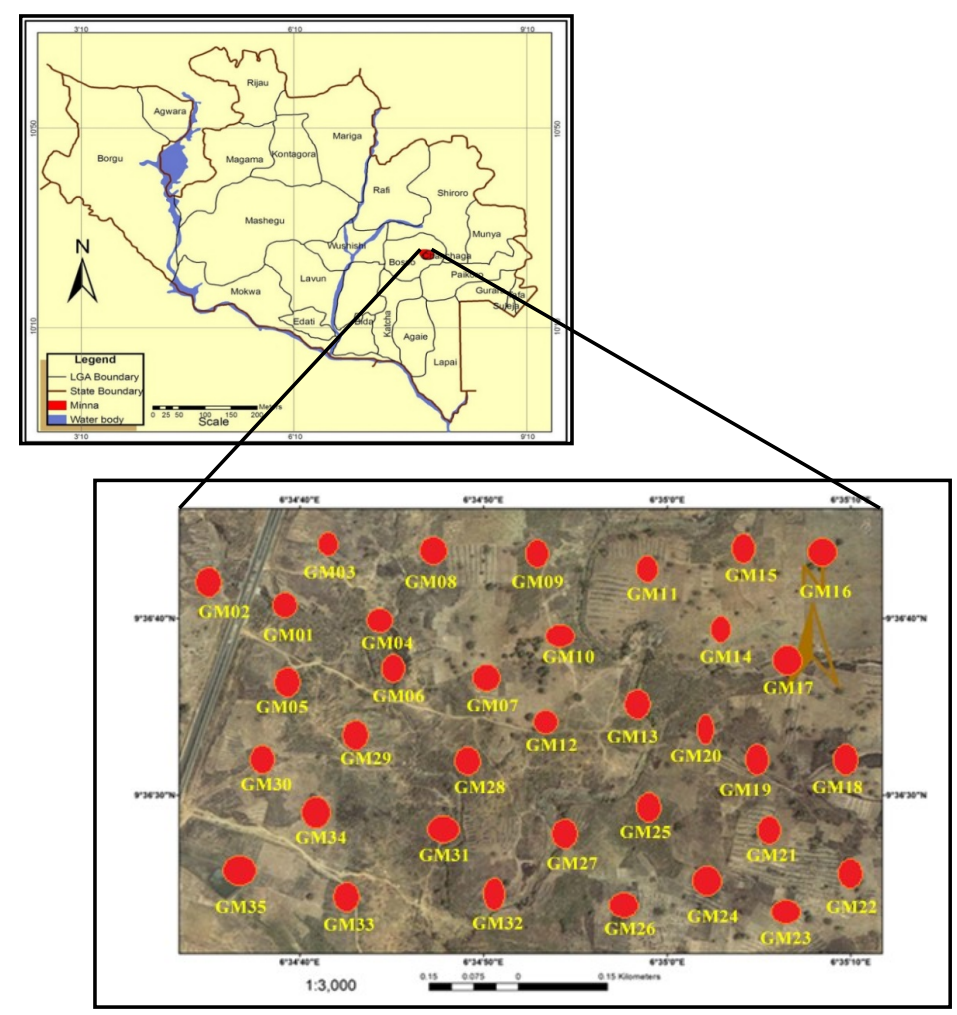

Fig. (1): Location map of the study area and the sampling points. 


\section{Sample Collection and Preparation}

40 surface soil samples were randomly collected at $15 \mathrm{~cm}$ of the upper soil surface from Gababiyu artisanal goldmine for radiometric analysis. The samples were carefully collected at different points to adequately represent the entire mine site. $1.0 \pm 0.1 \mathrm{~kg}$ of the collected soil samples were neatly packed into well labelled polyethylene bags (GM01 to GM40) and conveyed to the laboratory for preparation.

In order to assure completely moisture free samples, each soil sample was air dried at room temperature for 96 hours in the Applied Nuclear Physics Laboratory, Federal University of Technology Minna. The dried samples were thoroughly pulverized and sieved using BSS 63 aperture $(\approx 250$ micron mesh size) to obtain uniformly homogenous sample matrix with improved surface area to volume ratio. $500 \pm 0.1 \mathrm{~g}$ of the sieved samples were packed in accurately labeled polyethylene bags and transported for analysis to the Centre for Energy Research and Training (CERT), Ahmadu Bello University, Zaria. Before analysis, each sample was packaged into radon-impermeable cylindrical plastic containers, selected based on the volume of the detector vessel (7.6 $\mathrm{cm}$ by $7.6 \mathrm{~cm}$ ) geometry. Each container was triple sealed by smearing of the inner rim of container lid with Vaseline jelly, filling the lid assembly gap with candle wax to block the gaps between lid and con- tainer, and tight-sealing lid-container with masking adhesive tape. Sealed samples were stored for about 35 days to allow radon and its short-lived progenies attain secular radioactive equilibrium (Girigisu $\boldsymbol{e t}$ al., 2014) prior to gamma spectrometry analysis.

\section{Sample Analysis}

Gamma spectrometric analysis was carried out on the samples using a $7.62 \mathrm{~cm} \times 7.62 \mathrm{~cm} \mathrm{NaI}(\mathrm{Tl})$ gamma detector crystal optically coupled to a photomultiplier tube (PMT). The assembly has a preamplifier incorporated into it and a $1 \mathrm{KV}$ external source. The detector was enclosed in a $6 \mathrm{~cm}$ lead shield lined with cadmium and copper sheets to minimize the effects of background and scattered radiation. Data acquisition and gamma spectra analysis was performed using Maestro software (Canberra Nuclear Products). Calibration of the system for energy and efficiency were done prior to sample analysis using two calibration point sources: ${ }^{137} \mathrm{Cs}$ and ${ }^{60} \mathrm{Co}$, with amplifier gain of $72 \%$ energy resolution for the $661.16 \mathrm{KeV}$ of ${ }^{137} \mathrm{Cs}$. Dependability of calibration and quality assurance of gamma spectrometry were checked using the IAEA gamma spectrometric reference materials RGU-1, RGTh-1 and RGK-1. Characteristic gamma energy lines used to compute the specific activities of primordial radionuclides are given in Table 1.

Table (1): Detected radionuclides and their gamma energy lines used for determination of activity.

\begin{tabular}{|c|c|c|}
\hline Nuclide of interest & Detected isotope & $\gamma$-ray energy (keV) \\
\hline${ }^{226} \mathrm{Ra}$ & ${ }^{214} \mathrm{Bi}$ & 1764.0 \\
\hline${ }^{232} \mathrm{Th}$ & ${ }^{208} \mathrm{Tl}$ & 2614.5 \\
\hline${ }^{40} \mathrm{~K}$ & ${ }^{40} \mathrm{~K}$ & 1460.0 \\
\hline
\end{tabular}

The system was set at a working energy range of 0-3000 keV to accommodate the energy range of interest in the study. Each sample was counted for a period of 29000 seconds ( $\approx 8$ hours). The area of each energy peak in the spectrum was used to compute the activity concentrations in each sample by employing the equation (Njinga et al., 2015):

$$
A_{i}\left(B q ~ k g^{-1}\right)=\frac{C_{n}}{I_{(\gamma)} \varepsilon M T}
$$


where, $A_{i}$ is the activity concentration of a particular radionuclide in the sample, $\mathrm{C}_{\mathrm{n}}$ is the net count rate (counts per second), $\mathrm{I}_{(\gamma)}$ is the emission probability of a specific energy photo peak, $\varepsilon \mathcal{E}$ is the absolute efficiency of the detecting system, $\mathrm{T}$ is the time for collecting the sample spectrum, and $\mathrm{M}$ is the mass of the sample.

\section{Radiological Parameters}

From the activity concentrations obtained using Equation 1, the following assessment criteria were used to quantify exposure to radiation in the artisanal gold mining area.

\section{Radium Equivalent Activity ( $\left.R a_{e q}\right)$}

Radium Equivalent Activity $\left(\mathrm{Ra}_{\mathrm{eq}}\right)$ is the weighted sum of hazards associated with ${ }^{226} \mathrm{Ra},{ }^{232} \mathrm{Th}$ and ${ }^{40} \mathrm{~K}$. This index presumes that $1,0.7$ and $13 \mathrm{~Bq} / \mathrm{kg}$ of ${ }^{226} \mathrm{Ra},{ }^{232} \mathrm{Th}$ and ${ }^{40} \mathrm{~K}$, respectively, produce equal terrestrial gamma dose rates (Beretka \& Mathew, 1985; Kolo et al., 2019; UNSCEAR, 2000). UNSCEAR (2000) stipulates a threshold of $370 \mathrm{~Bq} / \mathrm{kg}$ for $\mathrm{Ra}_{\mathrm{eq}}$ (Suleiman et al., 2018). $\mathrm{Ra}_{\mathrm{eq}}$ was estimated using the equation (Kolo et al., 2019; Osimobi et al., 2018):

$$
\mathrm{Ra}_{\mathrm{eq}}=\mathrm{A}_{\mathrm{Ra}}+1.43 \mathrm{~A}_{\mathrm{Th}}+0.077 \mathrm{~A}_{\mathrm{K}}
$$

where $A_{R a}, A_{T h}$ and $A_{K}$ are the specific activity concentrations of ${ }^{226} \mathrm{Ra},{ }^{232} \mathrm{Th}$ and ${ }^{40} \mathrm{~K}$, respectively, in the soil samples.

\section{Gamma Radiation Dose $\left(D_{R}\right)$}

The gamma radiation dose or absorbed dose $\left(D_{R}\right)$ at $1 \mathrm{~m}$ above the ground was estimated using the equation (Munyaradzi et al., 2018; UNSCEAR, 2000):

$$
D_{R}=0.462 \mathrm{~A}_{\mathrm{Ra}}+0.604 \mathrm{~A}_{\mathrm{Th}}+0.0417 \mathrm{~A}_{\mathrm{K}}
$$

where $\mathrm{D}_{\mathrm{R}}$ is the gamma radiation dose in $\mathrm{nGy} / \mathrm{h}$ and the coefficients $(0.462,0.604$ and 0.0417 in $\mathrm{nGy} / \mathrm{h}$ per $\mathrm{Bq} / \mathrm{kg}$ ) are the dose conversion factors for ${ }^{226} \mathrm{Ra},{ }^{232} \mathrm{Th}$ and ${ }^{40} \mathrm{~K}$, respectively, as contained in the

\section{UNSCEAR (2008) report.}

\section{Annual Effective Dose Equivalent (AEDE)}

Annual Effective Dose Equivalent (AEDE) in $\mathrm{mSv} / \mathrm{y}$ is estimated as the product of the gamma radiation dose, $\mathrm{D}(\mathrm{nGy} / \mathrm{h})$, time in a year (8760 hours), dose conversion factor of $0.7 \mathrm{~Sv} / \mathrm{Gy}$ and occupancy factor of 0.2 for outdoor exposure (Taskin et al., 2009; UNSCEAR, 2000). AEDE was computed using the equation:

$$
\mathrm{AEDE}=\mathrm{D} \times 8760 \times 0.7 \times 0.2 \times 10^{-6}
$$

ICRP (2007) provided AEDE threshold of 1 $\mathrm{mSv} / \mathrm{y}$ for public exposure.

\section{Annual Gonadal Dose Equivalent (AGDE)}

The annual gonadal dose equivalent (AGDE) is a measure of the dose received by the gonads (gamete producing organs) of exposed population in a year (Kolo et al., 2015; Morsy et al., 2012):

$\operatorname{AGDE}\left(\mu \mathrm{Sv} \cdot \mathrm{y}^{-1}\right)=$

$$
3.09 \mathrm{~A}_{\mathrm{Ra}}+4.18 \mathrm{~A}_{\mathrm{Th}}+0.314 \mathrm{~A}_{\mathrm{K}}
$$

where $A_{R a}, A_{T h}$ and $A_{K}$ assume their respective definitions given before.

\section{Activity Utilization Index (AUI)}

Activity Utilization Index (AUI) is a parametric model used in determining NORM dose levels in the atmosphere from soil samples (Osimobi et al., 2018). AUI was calculated from the specific activities of ${ }^{226} \mathrm{Ra},{ }^{232} \mathrm{Th}$ and ${ }^{40} \mathrm{~K}$ in the sampled soils using the equation (Osimobi et al., 2018; Sivakumar et al., 2014):

$$
\mathrm{AUI}=\left(\frac{\mathrm{A}_{\mathrm{Ra}}}{50 \mathrm{~Bq} / \mathrm{Kg}}\right) f_{\mathrm{Ra}}+\left(\frac{\mathrm{A}_{\mathrm{Th}}}{50 \mathrm{~Bq} / \mathrm{Kg}}\right) f_{\mathrm{Th}}+\left(\frac{\mathrm{A}_{\mathrm{K}}}{500 \mathrm{~Bq} / \mathrm{Kg}}\right) f_{\mathrm{K}}
$$

where $f_{\mathrm{Ra}}, f_{\mathrm{Th}}$ and $f_{\mathrm{K}}$ having the numerical values of $0.462,0.604$ and 0.041 , respectively, represent fragmentary supplements of ${ }^{226} \mathrm{Ra},{ }^{232} \mathrm{Th}$ and ${ }^{40} \mathrm{~K}$ to the entire gamma dose (Chandrasekaran et al., 2014). 


\section{External and Internal Hazard Indices}

External hazard index $\left(\mathrm{H}_{\mathrm{ex}}\right)$ is a parameter used for evaluating external exposure to gamma radiation in air. The maximum allowed value for $\mathrm{H}_{\mathrm{ex}}$ is 1 , which corresponds to the upper limit of $\mathrm{Ra}_{\mathrm{eq}}(370 \mathrm{~Bq} /$ kg) (Stranden, 1976; Suleiman et al., 2018). Internal hazard index $\left(\mathrm{H}_{\mathrm{in}}\right)$, on the other hand, is a factor used to evaluate the hazardous effects of radon and its short lived progeny to the respiratory organs $\mathbf{( S u}-$ leiman et al., 2018). The threshold for $\mathrm{H}_{\text {in }}$ is also 1.

The external hazard index $\left(\mathrm{H}_{\mathrm{ex}}\right)$ and internal hazard index $\left(\mathrm{H}_{\text {in }}\right)$ were estimated using the equations (Berekta and Mathew, 1985; Osimobi et al., 2018):

$\mathrm{H}_{\mathrm{ex}}=\frac{\mathrm{A}_{\mathrm{Ra}}}{370}+\frac{\mathrm{A}_{\mathrm{Th}}}{259}+\frac{\mathrm{A}_{\mathrm{K}}}{4810} \leq 1$

$\mathrm{H}_{\mathrm{in}}=\frac{\mathrm{A}_{\mathrm{Ra}}}{185}+\frac{\mathrm{A}_{\mathrm{Th}}}{259}+\frac{\mathrm{A}_{\mathrm{K}}}{4810} \leq 1$

\section{Representative Gamma Index}

Representative gamma index $\left(\mathrm{I}_{\gamma \mathrm{r}}\right)$ is used to evaluate the conformity of soil to dose standards set for building materials (Jibiri et al., 2014; Kolo et al., 2019). It categorizes materials that may induce radiological risk if deployed for construction (Osimobi et al., 2018). $\mathrm{I}_{\gamma \mathrm{r}}$ was computed from the equation (Khater, et al., 2010; Osimobi et al., 2018)::

$I_{\gamma \mathrm{r}}=\frac{A_{\mathrm{Ra}}}{150}+\frac{A_{\mathrm{Th}}}{100}+\frac{A_{\mathrm{K}}}{1500}$

$\mathrm{I}_{\gamma \mathrm{r}}$ must be $\leq 1$ to satisfy the given dose criteria. This corresponds to an annual effective dose below 1 mSv (Kolo et al., 2019; Osimobi et al., 2018).

\section{Excess lifetime Cancer Risk (ELCR)}

Excess Lifetime Cancer Risk (ELCR) is a measure of the probability that a certain stochastic effect will occur in an individual exposed to low doses of ionizing radiation over a given period of time (Turyahabwa et al., 2016; UNSCEAR, 2000). The most common radiation induced health effects are incidence of cancers and genetic effects. ELCR was estimated using the equation (Munyaradzi et al., 2018; Taskin et al., 2009): :

\section{$\mathrm{ELCR}=\mathrm{AEDE} \times \mathrm{DL} \times \mathrm{RF}$}

where DL is the average duration of human life (estimated to be 70 years) and $\mathrm{RF}$ is risk factor $\left(\mathrm{Sv}^{-1}\right)$ or fatal cancer risk per sievert. For stochastic effects, which produce low background radiation, the ICRP 60 stipulates RF value of 0.05 for public exposure (Munyaradzi et al., 2018; Taskin et al., 2009).

\section{RESULTS}

Specific activities of primordial radionuclides in soil samples collected from Gababiyu artisanal goldmine along with the location coordinates are shown in Table 2. Activity concentrations of ${ }^{226} \mathrm{Ra}$ varied from $10.27 \pm 2.88 \mathrm{~Bq} / \mathrm{kg}$ to $152.60 \pm 3.80 \mathrm{~Bq} / \mathrm{kg}$ with an average value of $65.06 \pm 4.20 \mathrm{~Bq} / \mathrm{kg}$. Specific activity values for ${ }^{232} \mathrm{Th}$ ranged between $32.67 \pm 1.93$ $\mathrm{Bq} / \mathrm{kg}$ to $185.90 \pm 6.06 \mathrm{~Bq} / \mathrm{kg}$, with mean activity value of $87.63 \pm 2.90 \mathrm{~Bq} / \mathrm{kg}$. ${ }^{40} \mathrm{~K}$ show much higher activity values than ${ }^{226} \mathrm{Ra}$ and ${ }^{232} \mathrm{Th}$ which of course should be expected owing to the natural abundance of ${ }^{40} \mathrm{~K}$ in soils.

Activity concentration of ${ }^{40} \mathrm{~K}$ varied from $35.18 \pm 1.45 \mathrm{~Bq} / \mathrm{kg}$ to $947.50 \pm 7.51 \mathrm{~Bq} / \mathrm{kg}$, with mean value of $267.94 \pm 4.29 \mathrm{~Bq} / \mathrm{kg}$ (Table 2). The average activity value for ${ }^{40} \mathrm{~K}$ in the investigated mining site was lower than the world mean value of $400 \mathrm{~Bq} / \mathrm{kg}$.

\section{Radiological Parameters}

Computed $\mathrm{Ra}_{\mathrm{eq}}$, radiological doses and other radiation hazard indices are given in Table 3 . $\mathrm{Ra}_{\mathrm{eq}}$ varied from $96.67 \pm 4.56$ to $396.18 \pm 13.87 \mathrm{~Bq} \cdot \mathrm{kg}^{-1}$ with mean values of $210.86 \pm 8.66 \mathrm{~Bq} \cdot \mathrm{kg}^{-1}$. This value was below the global upper limit of $370 \mathrm{~Bq} \cdot \mathrm{kg}^{-1}$ (Kolo et al., 2012; UNSCEAR, 2000). $D_{R}$ at $1 \mathrm{~m}$ above the ground varied from $44.02 \mathrm{nGy} / \mathrm{h}$ to $173.46 \mathrm{nGy} / \mathrm{h}$, with an average value of $94.16 \mathrm{nGy} / \mathrm{h}$. 
Table (2): Activity concentrations of NORM in soil samples collected from Gababiyu artisanal goldmine.

\begin{tabular}{|c|c|c|c|c|c|}
\hline \multirow{2}{*}{ Sample ID } & \multicolumn{2}{|c|}{ Coordinates } & \multicolumn{3}{|c|}{ Activity concentration ( $\mathrm{Bq} / \mathrm{kg})$} \\
\hline & Longitude & Latitude & ${ }^{226} \mathbf{R a}$ & ${ }^{232} \mathrm{Th}$ & ${ }^{40} \mathbf{K}$ \\
\hline GM01 & $06^{\circ} 34^{\prime} 40.9^{\prime \prime}$ & $09^{\circ} 36^{\prime} 39.5^{\prime \prime}$ & $10.27 \pm 2.88$ & $60.12 \pm 0.31$ & $183.84 \pm 8.58$ \\
\hline GM02 & $06^{\circ} 34^{\prime} 40.4^{\prime \prime}$ & $09^{\circ} 36^{\prime} 38.2^{\prime \prime}$ & $129.1 \pm 3.40$ & $96.92 \pm 3.07$ & $236.02 \pm 1.56$ \\
\hline GM03 & $06^{\circ} 34^{\prime} 39.6^{\prime \prime}$ & $09^{\circ} 36^{\prime} 39.1^{\prime \prime}$ & $19.18 \pm 2.80$ & $48.21 \pm 0.94$ & $144.85 \pm 5.47$ \\
\hline GM04 & $06^{\circ} 34^{\prime} 40.0^{\prime \prime}$ & $09^{\circ} 36^{\prime} 39.8^{\prime \prime}$ & $89.13 \pm 6.75$ & $38.06 \pm 4.72$ & $137.82 \pm 1.14$ \\
\hline GM05 & $06^{\circ} 34^{\prime} 40.0^{\prime \prime}$ & $09^{\circ} 36^{\prime} 40.5^{\prime \prime}$ & $41.44 \pm 3.08$ & $80.80 \pm 0.28$ & $732.34 \pm 6.44$ \\
\hline GM06 & $06^{\circ} 34^{\prime} 40.2^{\prime \prime}$ & $09^{\circ} 36^{\prime} 40.4^{\prime \prime}$ & $17.50 \pm 2.24$ & $78.76 \pm 1.53$ & $277.74 \pm 7.94$ \\
\hline GM07 & $06^{\circ} 34^{\prime} 41.7^{\prime \prime}$ & $09^{\circ} 36^{\prime} 40.4^{\prime \prime}$ & $152.60 \pm 3.80$ & $124.72 \pm 1.34$ & $166.84 \pm 3.00$ \\
\hline GM08 & $06^{\circ} 34^{\prime} 42.7^{\prime \prime}$ & $09^{\circ} 36^{\prime} 40.1^{\prime \prime}$ & $81.07 \pm 2.84$ & $91.57 \pm 2.20$ & $281.65 \pm 6.38$ \\
\hline GM09 & $06^{\circ} 34^{\prime} 43.2^{\prime \prime}$ & $09^{\circ} 36^{\prime} 39.5^{\prime \prime}$ & $38.48 \pm 3.00$ & $66.33 \pm 1.34$ & $247.06 \pm 6.86$ \\
\hline GM10 & $06^{\circ} 34^{\prime} 43.3^{\prime \prime}$ & $09^{\circ} 36^{\prime} 38.6^{\prime \prime}$ & $120.19 \pm 4.99$ & $185.90 \pm 6.06$ & $135.41 \pm 2.90$ \\
\hline GM11 & $06^{\circ} 34^{\prime} 45.8^{\prime \prime}$ & $09^{\circ} 36^{\prime} 39.4^{\prime \prime}$ & $61.45 \pm 4.68$ & $59.88 \pm 2.08$ & $189.57 \pm 2.63$ \\
\hline GM12 & $06^{\circ} 34^{\prime} 45.4^{\prime \prime}$ & $09^{\circ} 36^{\prime} 40.4 "$ & $75.64 \pm 3.36$ & $96.41 \pm 1.42$ & $228.51 \pm 2.57$ \\
\hline GM13 & $06^{\circ} 34^{\prime} 44.2^{\prime \prime}$ & $09^{\circ} 36^{\prime} 39.5^{\prime \prime}$ & $25.05 \pm 0.92$ & $63.62 \pm 0.20$ & $233.34 \pm 8.85$ \\
\hline GM14 & $06^{\circ} 34^{\prime} 43.8^{\prime \prime}$ & $09^{\circ} 36^{\prime} 39.1^{\prime \prime}$ & $84.95 \pm 4.52$ & $83.75 \pm 1.02$ & $184.69 \pm 5.79$ \\
\hline GM15 & $06^{\circ} 34^{\prime} 44.2^{\prime \prime}$ & $09^{\circ} 36^{\prime} 38.9^{\prime \prime}$ & $28.33 \pm 1.08$ & $32.67 \pm 1.93$ & $281.65 \pm 9.49$ \\
\hline GM16 & $06^{\circ} 34^{\prime} 45.0^{\prime \prime}$ & $09^{\circ} 36^{\prime} 38.1^{\prime \prime}$ & $95.68 \pm 8.47$ & $66.80 \pm 7.67$ & $174.34 \pm 1.24$ \\
\hline GM17 & $06^{\circ} 34^{\prime} 42.9^{\prime \prime}$ & $09^{\circ} 36^{\prime} 36.8^{\prime \prime}$ & $89.90 \pm 3.32$ & $79.62 \pm 0.39$ & $291.20 \pm 3.49$ \\
\hline GM18 & $06^{\circ} 34^{\prime} 42.3^{\prime \prime}$ & $09^{\circ} 36^{\prime} 37.6^{\prime \prime}$ & $33.52 \pm 2.60$ & $60.43 \pm 2.01$ & $82.48 \pm 3.00$ \\
\hline GM19 & $06^{\circ} 34^{\prime} 42.5^{\prime \prime}$ & $09^{\circ} 36^{\prime} 37.6^{\prime \prime}$ & $51.06 \pm 3.80$ & $73.41 \pm 1.61$ & $232.26 \pm 5.68$ \\
\hline GM20 & $06^{\circ} 34^{\prime} 42.5^{\prime \prime}$ & $09^{\circ} 36^{\prime} 37.3^{\prime \prime}$ & $41.36 \pm 5.43$ & $109.23 \pm 1.85$ & $297.96 \pm 6.01$ \\
\hline GM21 & $06^{\circ} 34^{\prime} 53.6^{\prime \prime}$ & $09^{\circ} 36^{\prime} 31.0^{\prime \prime}$ & $92.40 \pm 7.79$ & $110.45 \pm 6.88$ & $333.66 \pm 1.00$ \\
\hline GM22 & $06^{\circ} 34^{\prime} 53.3^{\prime \prime}$ & $09^{\circ} 36^{\prime} 30.6^{\prime \prime}$ & $88.66 \pm 3.76$ & $75.77 \pm 0.83$ & $260.69 \pm 0.97$ \\
\hline GM23 & $06^{\circ} 34^{\prime} 50.9^{\prime \prime}$ & $09^{\circ} 36^{\prime} 31.2^{\prime \prime}$ & $41.71 \pm 4.00$ & $52.49 \pm 1.10$ & $35.18 \pm 1.45$ \\
\hline GM24 & $06^{\circ} 34^{\prime} 54.4^{\prime \prime}$ & $09^{\circ} 36^{\prime} 30.8^{\prime \prime}$ & $22.22 \pm 1.68$ & $68.34 \pm 1.42$ & $135.46 \pm 6.06$ \\
\hline GM25 & $06^{\circ} 34^{\prime} 53.9^{\prime \prime}$ & $09^{\circ} 36^{\prime} 31.2 ”$ & $108.42 \pm 4.43$ & $98.45 \pm 5.23$ & $157.34 \pm 0.72$ \\
\hline GM26 & $06^{\circ} 34^{\prime} 54.1^{\prime \prime}$ & $09^{\circ} 36^{\prime} 30.8^{\prime \prime}$ & $41.40 \pm 4.44$ & $83.00 \pm 2.24$ & $209.90 \pm 5.63$ \\
\hline GM27 & $06^{\circ} 34^{\prime} 53.5^{\prime \prime}$ & $09^{\circ} 36^{\prime} 30.7^{\prime \prime}$ & $97.32 \pm 5.51$ & $92.01 \pm 5.62$ & $162.05 \pm 1.20$ \\
\hline GM28 & $06^{\circ} 34^{\prime} 53.8^{\prime \prime}$ & $09^{\circ} 36^{\prime} 30.8^{\prime \prime}$ & $36.39 \pm 3.20$ & $45.85 \pm 4.76$ & $153.26 \pm 0.47$ \\
\hline GM29 & $06^{\circ} 34^{\prime} 53.8^{\prime \prime}$ & $09^{\circ} 36^{\prime} 31.0^{\prime \prime}$ & $102.91 \pm 7.95$ & $132.90 \pm 8.85$ & $232.20 \pm 1.56$ \\
\hline GM30 & $06^{\circ} 34^{\prime} 53.1^{\prime \prime}$ & $09^{\circ} 36^{\prime} 30.5^{\prime \prime}$ & $42.03 \pm 3.76$ & $100.46 \pm 0.24$ & $130.10 \pm 6.92$ \\
\hline GM31 & $06^{\circ} 34^{\prime} 53.6^{\prime \prime}$ & $09^{\circ} 36^{\prime} 31.6^{\prime \prime}$ & $34.52 \pm 2.00$ & $68.10 \pm 0.98$ & $168.77 \pm 6.76$ \\
\hline GM32 & $06^{\circ} 34^{\prime} 50.6^{\prime \prime}$ & $09^{\circ} 36^{\prime} 31.2^{\prime \prime}$ & $13.83 \pm 6.63$ & $99.75 \pm 0.24$ & $405.86 \pm 6.97$ \\
\hline GM33 & $06^{\circ} 34^{\prime} 53.8^{\prime \prime}$ & $09^{\circ} 36^{\prime} 31.4^{\prime \prime}$ & $99.79 \pm 7.79$ & $122.64 \pm 3.26$ & $322.55 \pm 1.06$ \\
\hline GM34 & $06^{\circ} 34^{\prime} 42.2^{\prime \prime}$ & $09^{\circ} 36^{\prime} 32.3^{\prime \prime}$ & $22.58 \pm 0.76$ & $61.18 \pm 0.39$ & $829.41 \pm 7.83$ \\
\hline GM35 & $06^{\circ} 34^{\prime} 43.2^{\prime \prime}$ & $09^{\circ} 36^{\prime} 32.0^{\prime \prime}$ & $116.79 \pm 2.88$ & $123.07 \pm 2.56$ & $93.90 \pm 0.21$ \\
\hline GM36 & $06^{\circ} 34^{\prime} 43.4^{\prime \prime}$ & $09^{\circ} 36^{\prime} 31.6^{\prime \prime}$ & $75.60 \pm 5.03$ & $137.18 \pm 4.95$ & $408.27 \pm 7.99$ \\
\hline GM37 & $06^{\circ} 34^{\prime} 42.7^{\prime \prime}$ & $09^{\circ} 36^{\prime} 32.1^{\prime \prime}$ & $83.61 \pm 7.47$ & $83.08 \pm 9.24$ & $91.06 \pm 0.39$ \\
\hline GM38 & $06^{\circ} 34^{\prime} 41.3^{\prime \prime}$ & $09^{\circ} 36^{\prime} 29.9^{\prime \prime}$ & $49.07 \pm 4.24$ & $165.69 \pm 6.68$ & $947.50 \pm 7.51$ \\
\hline GM39 & $06^{\circ} 34^{\prime} 42.0^{\prime \prime}$ & $09^{\circ} 36^{\prime} 32.0^{\prime \prime}$ & $109.58 \pm 5.23$ & $87.41 \pm 5.15$ & $155.62 \pm 1.02$ \\
\hline GM40 & $06^{\circ} 34^{\prime} 41.8^{\prime \prime}$ & $09^{\circ} 36^{\prime} 30.7^{\prime \prime}$ & $37.56 \pm 5.47$ & $100.30 \pm 3.07$ & $745.27 \pm 6.97$ \\
\hline Min. & & & $10.27 \pm 2.88$ & $32.67 \pm 1.93$ & $35.18 \pm 1.45$ \\
\hline Max. & & & $152.60 \pm 3.80$ & $185.90 \pm 6.06$ & $947.50 \pm 7.51$ \\
\hline Mean & & & $65.06 \pm 4.20$ & $87.63 \pm 2.89$ & $267.94 \pm 4.29$ \\
\hline
\end{tabular}


Table (3) : Radiological parameters characterizing the soil samples collected from Gababiyu artisanal goldmine.

\begin{tabular}{|c|c|c|c|c|c|c|c|c|c|}
\hline \multirow{2}{*}{$\begin{array}{c}\text { Sample } \\
\text { ID }\end{array}$} & \multirow{2}{*}{$\begin{array}{c}\mathbf{R a}_{\text {eq }} \\
(\mathbf{B q} / \mathbf{K g})\end{array}$} & \multicolumn{3}{|c|}{ Radiological dose } & \multicolumn{4}{|c|}{ Radiation hazard indices $(\leq 1)$} & \multirow{2}{*}{$\begin{array}{l}\text { ELCR } \\
\left(\times 10^{-3}\right)\end{array}$} \\
\hline & & $D_{R}(n G y / h)$ & $\operatorname{AEDE}(\mathrm{mSv} / \mathrm{y})$ & $\operatorname{AGDE}(\mu \mathrm{Sv} / \mathrm{y})$ & AUI & $\mathbf{H}_{\mathrm{ex}}$ & $\mathbf{H}_{\text {in }}$ & $\mathbf{I}_{\gamma \mathrm{r}}$ & \\
\hline GM01 & $110.29 \pm 3.99$ & 48.72 & 0.06 & 340.75 & 0.84 & 0.30 & 0.33 & 0.79 & 0.21 \\
\hline GM02 & $285.71 \pm 7.90$ & 128.03 & 0.16 & 878.16 & 2.38 & 0.77 & 1.12 & 1.99 & 0.55 \\
\hline GM03 & $99.19 \pm 4.57$ & 44.02 & 0.05 & 306.24 & 0.77 & 0.27 & 0.32 & 0.71 & 0.19 \\
\hline GM04 & $154.10 \pm 13.58$ & 69.91 & 0.09 & 477.77 & 1.29 & 0.42 & 0.66 & 1.07 & 0.30 \\
\hline GM05 & $213.20 \pm 3.96$ & 98.49 & 0.12 & 695.74 & 1.42 & 0.58 & 0.69 & 1.57 & 0.42 \\
\hline GM06 & $151.37 \pm 5.04$ & 67.24 & 0.08 & 470.49 & 1.14 & 0.41 & 0.46 & 1.09 & 0.29 \\
\hline GM07 & $343.60 \pm 5.94$ & 152.79 & 0.19 & 1045.24 & 2.93 & 0.93 & 1.34 & 2.38 & 0.66 \\
\hline GM08 & $233.56 \pm 6.47$ & 104.51 & 0.13 & 721.73 & 1.88 & 0.63 & 0.85 & 1.64 & 0.45 \\
\hline GM09 & $152.24 \pm 5.43$ & 68.14 & 0.08 & 473.74 & 1.18 & 0.41 & 0.52 & 1.08 & 0.29 \\
\hline GM10 & $396.18 \pm 13.87$ & 173.46 & 0.21 & 1190.97 & 3.37 & 1.07 & 1.40 & 2.75 & 0.74 \\
\hline GM11 & $161.58 \pm 7.85$ & 72.47 & 0.09 & 499.73 & 1.31 & 0.44 & 0.60 & 1.13 & 0.31 \\
\hline GM12 & $230.94 \pm 5.58$ & 102.71 & 0.13 & 708.47 & 1.88 & 0.62 & 0.83 & 1.62 & 0.44 \\
\hline GM13 & $133.88 \pm 1.88$ & 59.73 & 0.07 & 416.60 & 1.02 & 0.36 & 0.43 & 0.96 & 0.26 \\
\hline GM14 & $218.80 \pm 6.42$ & 97.53 & 0.12 & 670.56 & 1.81 & 0.59 & 0.82 & 1.53 & 0.42 \\
\hline GM15 & $96.67 \pm 4.56$ & 44.57 & 0.05 & 312.55 & 0.68 & 0.26 & 0.34 & 0.70 & 0.19 \\
\hline GM16 & $204.52 \pm 19.52$ & 91.82 & 0.11 & 629.62 & 1.71 & 0.55 & 0.81 & 1.42 & 0.39 \\
\hline GM17 & $226.05 \pm 4.15$ & 101.77 & 0.12 & 702.05 & 1.82 & 0.61 & 0.85 & 1.59 & 0.44 \\
\hline GM18 & $126.20 \pm 5.69$ & 55.43 & 0.07 & 382.10 & 1.05 & 0.34 & 0.43 & 0.88 & 0.24 \\
\hline GM19 & $173.80 \pm 6.54$ & 77.62 & 0.10 & 537.57 & 1.38 & 0.47 & 0.61 & 1.23 & 0.33 \\
\hline GM20 & $220.32 \pm 8.54$ & 97.50 & 0.12 & 677.92 & 1.73 & 0.60 & 0.71 & 1.57 & 0.42 \\
\hline GM21 & $275.85 \pm 17.70$ & 123.31 & 0.15 & 851.96 & 2.22 & 0.75 & 1.00 & 1.94 & 0.53 \\
\hline GM22 & $216.96 \pm 5.01$ & 97.60 & 0.12 & 672.54 & 1.76 & 0.59 & 0.83 & 1.52 & 0.42 \\
\hline GM23 & $119.41 \pm 5.68$ & 52.44 & 0.06 & 359.36 & 1.02 & 0.32 & 0.44 & 0.83 & 0.23 \\
\hline GM24 & $130.26 \pm 4.17$ & 57.19 & 0.07 & 396.83 & 1.04 & 0.35 & 0.41 & 0.92 & 0.25 \\
\hline GM25 & $261.17 \pm 11.96$ & 116.12 & 0.14 & 795.97 & 2.20 & 0.71 & 1.00 & 1.81 & 0.50 \\
\hline GM26 & $176.12 \pm 8.07$ & 78.01 & 0.10 & 540.77 & 1.40 & 0.48 & 0.59 & 1.25 & 0.33 \\
\hline GM27 & $241.22 \pm 13.64$ & 107.29 & 0.13 & 736.18 & 2.02 & 0.65 & 0.91 & 1.68 & 0.46 \\
\hline GM28 & $113.68 \pm 10.03$ & 50.90 & 0.06 & 352.22 & 0.90 & 0.31 & 0.41 & 0.80 & 0.22 \\
\hline GM29 & $310.62 \pm 20.71$ & 137.50 & 0.17 & 946.41 & 2.58 & 0.84 & 1.12 & 2.17 & 0.59 \\
\hline GM30 & $195.56 \pm 4.63$ & 85.52 & 0.10 & 590.66 & 1.61 & 0.53 & 0.64 & 1.37 & 0.37 \\
\hline GM31 & $144.79 \pm 3.92$ & 64.12 & 0.08 & 444.33 & 1.16 & 0.39 & 0.48 & 1.02 & 0.28 \\
\hline GM32 & $187.55 \pm 7.51$ & 83.56 & 0.10 & 587.12 & 1.37 & 0.51 & 0.54 & 1.36 & 0.36 \\
\hline GM33 & $299.80 \pm 12.53$ & 133.63 & 0.16 & 922.26 & 2.43 & 0.81 & 1.08 & 2.11 & 0.57 \\
\hline GM34 & $173.78 \pm 1.92$ & 81.97 & 0.10 & 585.93 & 1.02 & 0.47 & 0.53 & 1.32 & 0.35 \\
\hline GM35 & $299.83 \pm 6.54$ & 132.21 & 0.16 & 904.80 & 2.57 & 0.81 & 1.13 & 2.07 & 0.57 \\
\hline GM36 & $302.98 \pm 12.73$ & 134.81 & 0.17 & 935.22 & 2.39 & 0.82 & 1.02 & 2.15 & 0.58 \\
\hline GM37 & $209.30 \pm 20.70$ & 92.61 & 0.11 & 634.23 & 1.78 & 0.57 & 0.79 & 1.45 & 0.40 \\
\hline GM38 & $358.65 \pm 14.36$ & 162.26 & 0.20 & 1141.72 & 2.53 & 0.97 & 1.10 & 2.62 & 0.70 \\
\hline GM39 & $246.42 \pm 12.67$ & 109.91 & 0.13 & 752.82 & 2.08 & 0.67 & 0.96 & 1.71 & 0.47 \\
\hline GM40 & $238.18 \pm 10.39$ & 109.01 & 0.13 & 769.34 & 1.62 & 0.64 & 0.75 & 1.75 & 0.47 \\
\hline Min. & $96.67 \pm 4.56$ & 44.02 & 0.05 & 306.24 & 0.68 & 0.26 & 0.32 & 0.70 & 0.19 \\
\hline Max. & $396.18 \pm 13.87$ & 173.46 & 0.21 & 1190.97 & 3.37 & 1.07 & 1.40 & 2.75 & 0.74 \\
\hline Mean & $210.86 \pm 8.66$ & 94.16 & 0.12 & 651.47 & 1.68 & 0.57 & 0.75 & 1.49 & 0.40 \\
\hline
\end{tabular}


Calculated AEDE values ranged between 0.05 $\mathrm{mSv} / \mathrm{y}$ and $0.21 \mathrm{mSv} / \mathrm{y}$, with mean value of 0.12 $\mathrm{mSv} / \mathrm{y}$, which was lower than the $1 \mathrm{mSv} / \mathrm{y}$ threshold recommended by ICRP (2007) for public exposure. AGDE recorded values ranging from $306.24 \mu \mathrm{Sv} / \mathrm{y}$ to $1190.97 \mu \mathrm{Sv} / \mathrm{y}$, with mean value of $651.47 \mu \mathrm{Sv} / \mathrm{y}$ Furthermore, the values computed for AUI ranged between 0.68 and 3.37, with a mean of 1.68. This satisfied the $<2$ threshold, corresponding to AEDE below $1 \mathrm{mSv} / \mathrm{y}$ for radiological safety (Osimobi $\boldsymbol{e t}$ al., 2018; Sivakumar et al., 2014). Computed values for external hazard index $\mathrm{H}_{\mathrm{ex}}$ and internal hazard index, $\mathrm{H}_{\mathrm{in}}$ ranged from 0.26 to 1.07 and 0.32 to 1.40 respectively, with average values of 0.57 and 0.75 in sequence. Furthermore, calculated values for $\mathrm{I}_{\gamma \mathrm{r}}$ varied from 0.70 to 2.75 , with about 30 of the investigated samples registering $I_{\gamma \mathrm{r}}$ values above unity. The computed mean $\mathrm{I}_{\gamma \mathrm{r}}$ of 1.49 was slightly above the global screening value of unity for building materials (Osimobi et al., 2018; Sivakumar et al., 2014). Similarly, computed ELCR values for the artisanal gold mining site varied from $0.19 \times 10^{-3}$ to $0.74 \times 10^{-3}$, with average value of $0.40 \times 10^{-3}$.

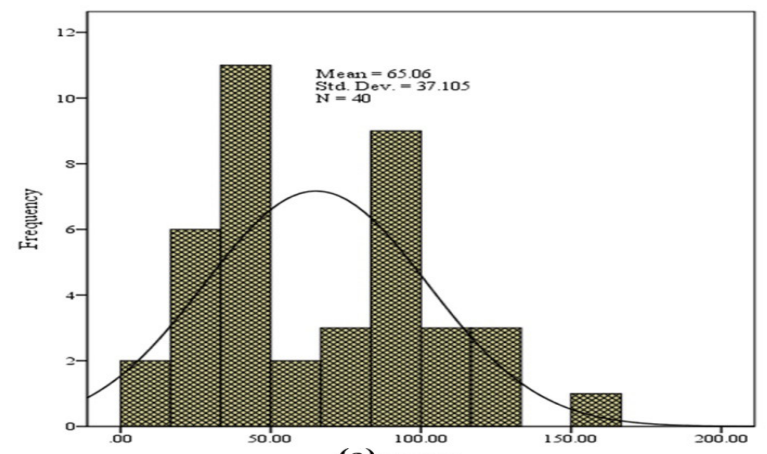

(a) $\operatorname{Ra}_{\mathbf{a}}-226$

\section{DISCUSSIONS}

Results presented in Table 2 clearly showed spatial variations in activity concentrations which, according to El-Mamney and Khater (2004) and Kolo et al. (2015), may be a result of geochemical and physiochemical characteristics of the radionuclides. However, despite the variations in the activity values, there appeared to be an even distribution of primordial radionuclides across the mining site as depicted in the frequency distribution histograms shown in Figure 2.

Mean activity concentration of ${ }^{226} \mathrm{Ra}$ was $65.06 \pm 4.20 \mathrm{~Bq} / \mathrm{kg}$, while ${ }^{232} \mathrm{Th}$ recorded average specific activity of $87.63 \pm 2.90 \mathrm{~Bq} / \mathrm{kg}$. These values were found to be higher than their respective global average of $35 \mathrm{~Bq} / \mathrm{kg}$ and $30 \mathrm{~Bq} / \mathrm{kg}$, respectively, as documented by UNSCEAR (2000) for normal soils. This therefore, points to the likelihood of radioactive pollution of Gababiyyu gold mining environment as a result of the constant and continuous mining activities performed by the locals.

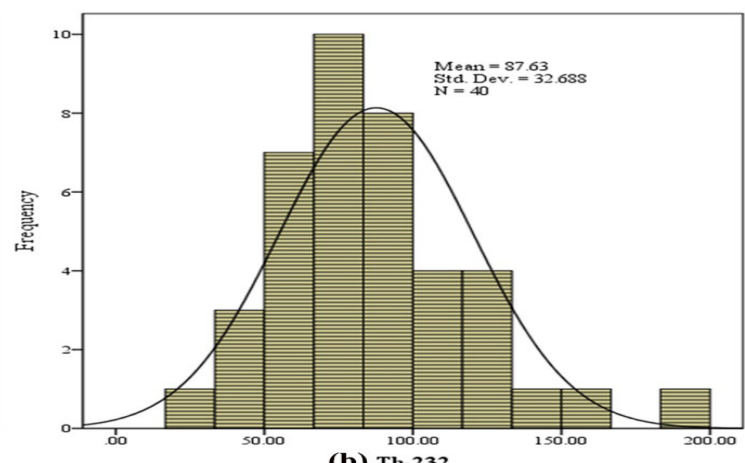

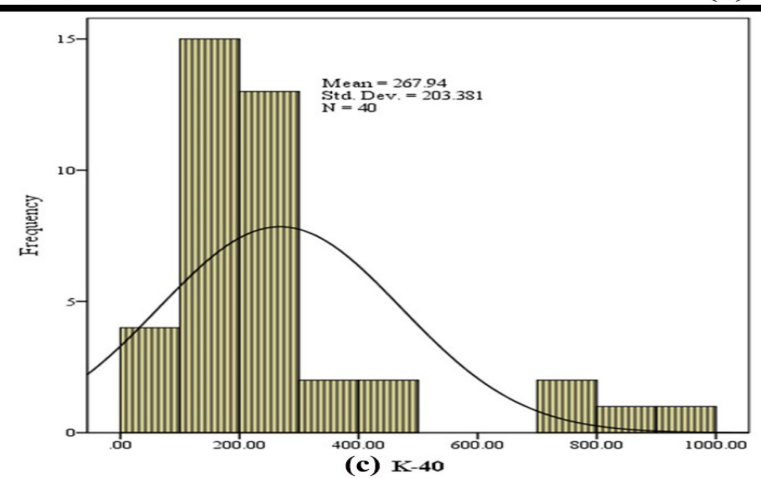

Fig. (2): Frequency distribution histograms of (a) ${ }^{226} \mathrm{Ra},(\mathrm{b}){ }^{232} \mathrm{Th}$ and $(c){ }^{40} \mathrm{~K}$ in the studied soil samples. 
Comparison of the data generated from this study with those of similar studies conducted in some parts of the world as presented in Table 4, showed some degree of agreement.

Table (4) : Comparison of specific activities (Bq/ $\mathrm{kg}$ ) of primordial radionuclides in the present study with similar studies around the world.

\begin{tabular}{|c|c|c|c|c|}
\hline Location & ${ }^{226} \mathrm{Ra}$ & ${ }^{232} \mathrm{Th}$ & ${ }^{40} \mathrm{~K}$ & Reference \\
\hline Nigeria & 49.43 & 37.69 & 564.63 & Suleiman et al. (2018) \\
\hline Uganda & 55.3 & 216 & 566.93 & Turyahabwa et al. (2016) \\
\hline China & 12.64 & 15.89 & 746.84 & Hu et al. (2016) \\
\hline Ghana & 65.1 & 71.8 & 1168.3 & Faanu et al. (2016) \\
\hline Nigeria & 55.3 & 26.4 & 505.1 & Ademola and Onyema (2014) \\
\hline Iraq & 77.33 & 9.36 & 426.31 & Al-Gazaly et al. (2014) \\
\hline India & 138.24 & 83.15 & 343.2 & Vinay Kumar Reddy et al. (2012) \\
\hline Malaysia & 178 & 841 & 104 & Kuan et al. (2009) \\
\hline Nigeria & 65.06 & 87.63 & 267.94 & Present study \\
\hline World average & 35 & 30 & 400 & UNSCEAR (2000) \\
\hline
\end{tabular}

Activity ratios of primordial radioisotopes were further computed in order to give a clear insight into the level of radioactive contamination of the investigated site. The plots of computed radioactivity ratios are shown in Figure 3. Computed ${ }^{232} \mathrm{Th}:{ }^{226} \mathrm{Ra}$ varied from 0.43 t0 7.22, with a mean value of 1.87 . This value for the investigated site was above the value for normal soils (1.2) reported by Eisenbud and Gesell (1997), which showed relative abundance of ${ }^{232} \mathrm{Th}$ compared to ${ }^{226} \mathrm{Ra}$ in the site. Average values for ${ }^{232} \mathrm{Th}:{ }^{40} \mathrm{~K}$ and ${ }^{226} \mathrm{Ra}:{ }^{40} \mathrm{~K}$ ratios were 0.46 and 0.37 , respectively. These values were lower than 1 , showing that ${ }^{40} \mathrm{~K}$ exhibits higher radioactivity compared to ${ }^{226} \mathrm{Ra}$ and ${ }^{232} \mathrm{Th}$ in the investigated mining site.

Gamma radiation dose $D_{R}$ characterizing the studied soil samples from Gababiyu artisanal mining site is shown in Figure 4. Average $D_{R}$ at $1 \mathrm{~m}$ above the ground was found to be $94.16 \mathrm{nGy} / \mathrm{h}$. Although this value appears to be relatively above the global average of $57 \mathrm{nG} / \mathrm{h}$ documented by UNSCEAR (2000), it compares moderately with results of similar studies around the world (Doyi et al., 2013; Kamunda et al., 2016; Kuan et al., 2009; Turyahabwa et al., 2016; Vinay Kumar Reddy et al., 2012).

Calculated $\mathrm{H}_{\mathrm{ex}}$ for the studied samples varied between 0.27 and 1.07, with a mean value of 0.57 . Although the average value was lower than the UNSCEAR established threshold of unity, one of the investigated samples (sample GM10) exceeded the threshold by about $7 \%$ as seen in Figure 5. This may however not constitute any immediate radiological threat that will require urgent attention.

A plot of the computed $\mathrm{H}_{\text {in }}$ for the studied soil samples is shown in Figure 6. Although the calculated mean $\mathrm{H}_{\text {in }}$ value of 0.75 was below the UNSCEAR threshold of unity, 8 of the samples exceeded this limit as can be seen in Figure 6. These include samples GM2, GM7, GM10, GM29, GM33, GM35, GM36 and GM38. This result points to the possibility of internal contamination of the local miners by radon and its byproducts after long time exposure. Radiation protection protocols must therefore be strictly adhered to by the miners at these locations for their radiation safety. 
The variation in the representative gamma index $I_{\gamma r}$ obtained for the studied samples is shown in Figure 7. 32 samples which represent about 80\% of the studied samples recorded $\mathrm{I}_{\gamma \mathrm{r}}$ values above the recommended UNSCEAR threshold. This shows that the investigated samples may induce radiation risk among the populace especially when used as aggregate of building material. Furthermore, strong positive correlation was found to exist between $\mathrm{I}_{\gamma \mathrm{r}}$ and ${ }^{226} \mathrm{Ra}(+0.72)$ and also with ${ }^{232} \mathrm{Th}(+0.93)$ as seen in Table 5. This showed that the rise in values of gamma index is principally due to ${ }^{226} \mathrm{Ra}$ and ${ }^{232} \mathrm{Th}$ contents in the studied samples. Thus, very serious precautionary measures must be taken in deploying soil from Gababiyu gold mining site for construction, from point of view of radiation protection.
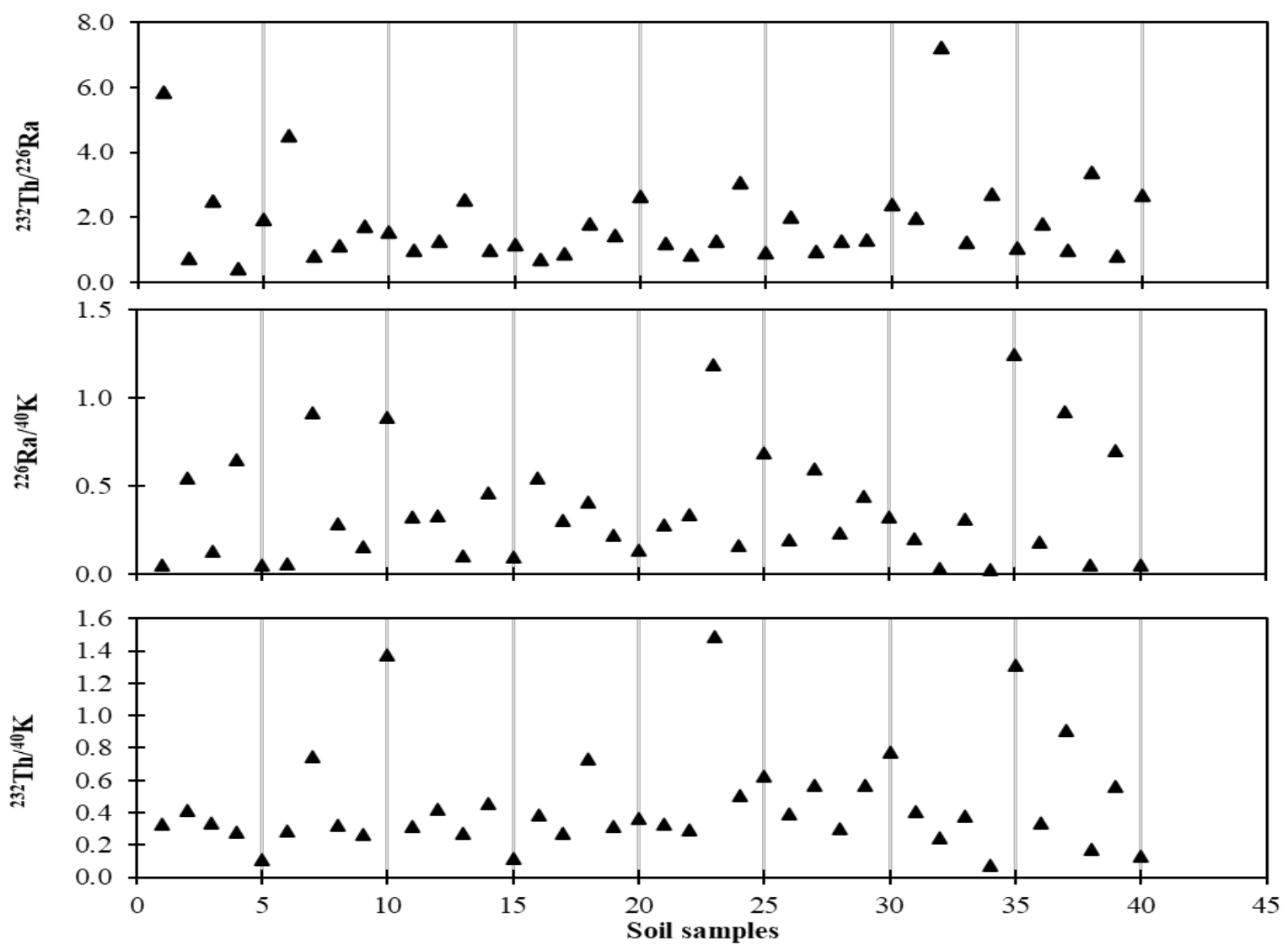

Fig. (3): Activity ratios of ${ }^{232} \mathrm{Th}^{226} \mathrm{Ra},{ }^{226} \mathrm{Ra}{ }^{40} \mathrm{~K}$ and ${ }^{232} \mathrm{Th} /{ }^{40} \mathrm{~K}$ in the studied soil samples.

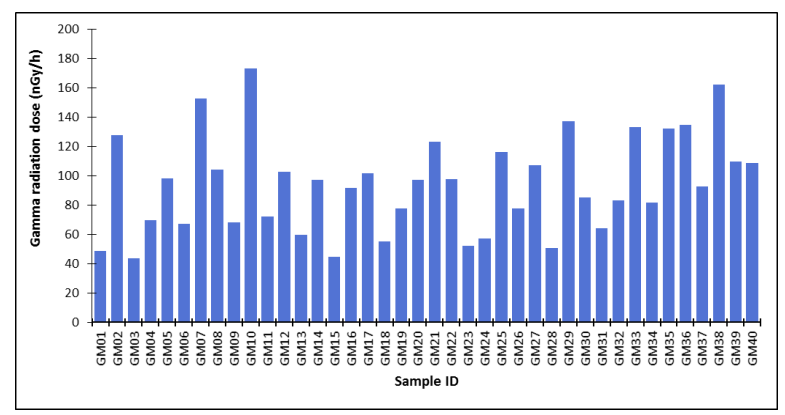

Fig. (4): Gamma Radiation Dose characterizing the considered samples.

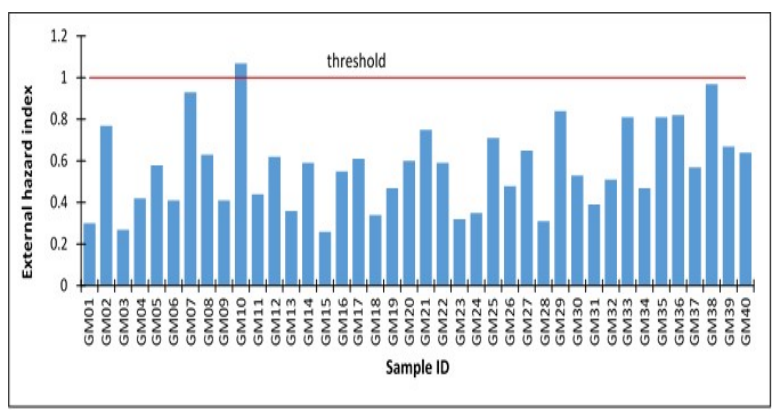

Fig. (5): External Hazard Index values obtained for the considered samples. 


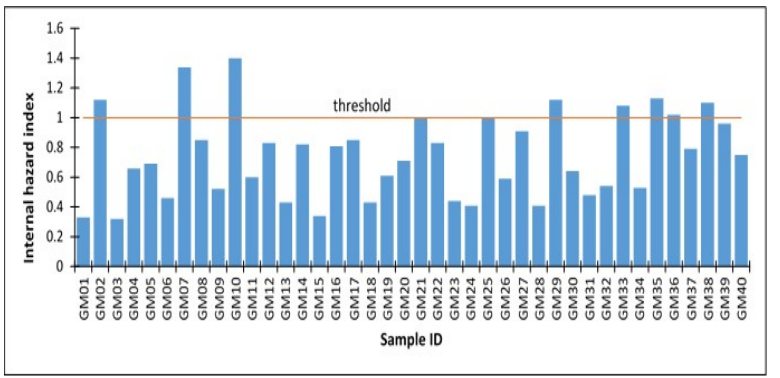

Fig. (6): Internal Hazard Index values obtained for the considered samples.

Computed average for ELCR was $0.40 \times 10^{-3}$, which is higher than the global mean of $0.29 \times 10^{-3}$ (Munyaradzi et al., 2018; Taskin et al., 2009). The results suggest that serious caution should be applied when using the soil from this gold mine as aggregates of building material or for agricultural purposes, in order to forestall likelihood of cancer incidence over long period of time.

\section{Pearson's Correlation Analysis}

To check the possibility of correlations and degree of dependency between primordial radionuclides and the computed radiological parameters, data obtained in this study were subjected to Pearson's correlation analysis. Computed linear correlation coefficients obtained at alpha testing level $\mathrm{p}<0.05$ for samples ( $\mathrm{n}=40$ ), were classified as "very

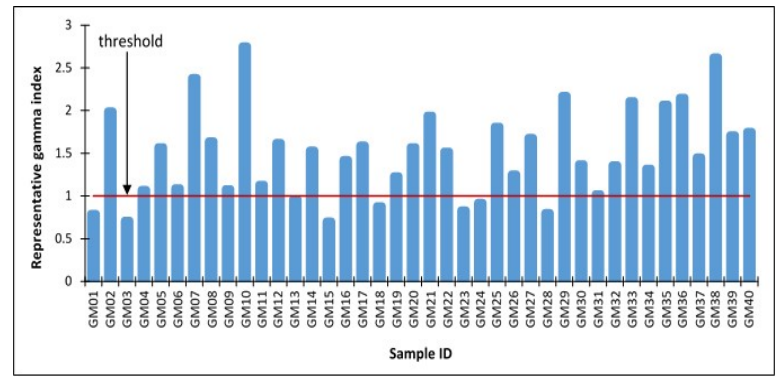

Fig. (7): Representative Gamma Index characterizing the considered samples.

strong" $(\mathrm{r}>0.75)$, "strong" $(0.50<\mathrm{r}<0.75)$, "weak" $(0.36<\mathrm{r}<0.49)$ and "very weak" $(\mathrm{r}<0.36)$, respectively. Results of the correlation analysis are presented in Table 5.

The results showed strong positive association between ${ }^{226} \mathrm{Ra}$ and ${ }^{232} \mathrm{Th}(\mathrm{r}=0.50)$, very weak negative relationship between ${ }^{226} \mathrm{Ra}$ and ${ }^{40} \mathrm{~K}(\mathrm{r}=-0.25)$ and very weak relationship between ${ }^{232} \mathrm{Th}$ and ${ }^{40} \mathrm{~K}(\mathrm{r}$ $=0.26$ ). This showed that ${ }^{226} \mathrm{Ra}$ and ${ }^{232} \mathrm{Th}$ may have a common natural origin and existence, different from that of ${ }^{40} \mathrm{~K}$. Very strong relationship was also noticed among all computed radiological variables ( $r>0.75$ ). Additionally, ${ }^{226} \mathrm{Ra}$ and ${ }^{232} \mathrm{Th}$ correlated very strongly $(r>0.75)$ with all computed radiological parameters (Table 5). This indicated that ${ }^{226} \mathrm{Ra}$ and ${ }^{232} \mathrm{Th}$ are the principal radionuclides responsible for gamma radiation emission in Gadabiyyu gold mining site.

Table (5) : Comparison of specific activities (Bq/ $\mathrm{kg}$ ) of primordial radionuclides in the present study with similar studies around the world.

\begin{tabular}{|c|c|c|c|c|c|c|c|c|c|c|c|c|}
\hline Variables & ${ }^{226} \mathbf{R a}$ & ${ }^{232} \mathbf{T h}$ & ${ }^{40} \mathbf{K}$ & $\mathbf{R a}_{\mathbf{e q}}$ & $\mathbf{D}_{\mathbf{R}}$ & AEDE & AGDE & AUI & $\mathbf{H}_{\mathrm{ex}}$ & $\mathbf{H}_{\text {in }}$ & $\mathbf{I}_{\gamma}$ & $\mathbf{E L C R}$ \\
\hline${ }^{226} \mathrm{Ra}$ & 1.00 & & & & & & & & & & & \\
\hline${ }^{232} \mathrm{Th}$ & 0.50 & 1.00 & & & & & & & & & & \\
\hline${ }^{40} \mathrm{~K}$ & -0.25 & 0.26 & 1.00 & & & & & & & & & \\
\hline $\mathrm{Ra}_{\mathrm{eq}}$ & 0.75 & 0.92 & 0.25 & 1.00 & & & & & & & & \\
\hline $\mathrm{D}_{\mathrm{R}}$ & 0.75 & 0.92 & 0.28 & 1.00 & 1.00 & & & & & & & \\
\hline $\mathrm{AEDE}$ & 0.75 & 0.92 & 0.28 & 1.00 & 1.00 & 1.00 & & & & & & \\
\hline $\mathrm{AGDE}$ & 0.73 & 0.92 & 0.31 & 1.00 & 1.00 & 1.00 & 1.00 & & & & & \\
\hline $\mathrm{AUI}$ & 0.84 & 0.89 & 0.05 & 0.98 & 0.97 & 0.97 & 0.97 & 1.00 & & & & \\
\hline $\mathrm{H}_{\mathrm{ex}}$ & 0.75 & 0.92 & 0.25 & 1.00 & 1.00 & 1.00 & 1.00 & 0.98 & 1.00 & & & \\
\hline $\mathrm{H}_{\mathrm{in}}$ & 0.89 & 0.83 & 0.09 & 0.97 & 0.97 & 0.97 & 0.96 & 0.99 & 0.97 & 1.00 & & \\
\hline $\mathrm{I}_{\gamma}$ & 0.72 & 0.93 & 0.30 & 1.00 & 1.00 & 1.00 & 1.00 & 0.97 & 1.00 & 0.96 & 1.00 & \\
\hline $\mathrm{ELCR}$ & 0.75 & 0.91 & 0.28 & 1.00 & 1.00 & 1.00 & 1.00 & 0.97 & 1.00 & 0.97 & 1.00 & 1.00 \\
\hline
\end{tabular}




\section{CONCLUSIONS}

Surface soil samples from artisanal gold mining environment in Gababiyu were assessed for their radiological contents using gamma spectrometry technique. Average specific activity values for ${ }^{226} \mathrm{Ra}$, ${ }^{232} \mathrm{Th}$ and ${ }^{40} \mathrm{~K}$ were $65.06 \mathrm{~Bq} / \mathrm{kg}, 87.63 \mathrm{~Bq} / \mathrm{kg}$ and $267.94 \mathrm{~Bq} / \mathrm{kg}$, respectively. These values, except for ${ }^{40} \mathrm{~K}$, were above the global mean values recommended by the United Nations Scientific Committee on Effects of Atomic Radiation. This gives an indication of possible radioactive pollution as a result of the local gold mining activities.

Average gamma dose rate was found to be slightly higher than the recommended global average. Some samples also showed slightly enhanced values of internal hazard index, which points to the possibility of internal contamination of the local miners by radon and its byproducts. Similarly, almost $80 \%$ of the studied samples recorded $\mathrm{I}_{\gamma \mathrm{r}}$ values above the recommended UNSCEAR threshold. This give an indication of the likelihood of occurrence of radiation incidences after long time exposure. Mean Excess Lifetime Cancer Risk of 0.40×10${ }^{3}$ may suggest that serious caution should be applied when using soil from Gababiyu artisanal gold mine as aggregates of building material or for agricultural purposes, in order to forestall likelihood of cancer incidence over long period of time. There is therefore the need for constant and continuous radiological monitoring of Gababiyu artisanal gold mine so as to keep any foreseeable radiation effects as low as reasonably achievable (ALARA), within the framework of social and economic provisions.

\section{ACKNOWLEDGEMENTS}

The authors wish to acknowledge the assistance and technical contributions of the staff of Centre for Energy Research and Training (CERT), Ahmadu Bello University, Zaria, Kaduna State, Nigeria.

\section{REFERENCES}

- Ademola, J.A. and Onyema, U.C. (2014): Assessment of Natural Radionuclides in Fly Ash Produced at Orji River Thermal Power Station, Nigeria and the Associated Radiological Impact. Natural Science, 2014.

- Ahmed, A.; Alhassan, D.U.; Shehu, J.; Alkali, A.; Alabi, O.O. and Mohammed, A. (2019): Geoelectrical Assessment of Aquifer Potentials and its Vulnerability to Contaminant at El-Amin Proposed University Site, Minna, Niger State, Nigeria. J. Waste Manag. \& Xenobiot., 2(2): 121.

- Ako, T.; Onoduku, U.; Oke, S.; Adamu, I.; Ali, S.; Mamodu, A. and Ibrahim, A. (2014): Environmental impact of artisanal gold mining in Luku, Minna, Niger state, North Central Nigeria. J. Geosci. \& Geomat., 2(1): 28.

- Al-Gazaly, H.; Al-Ulum, M.; Al-Hamidawi, A. and Al-Abbasi, A. (2014): Natural radioactivity in soil at regions around the uranium mine in Abu-Skhair Najaf Province, Iraq. Adv. Appl. Sci. Res., 5(1): 13.

- Alharbi, S.H. (2016): Measurements and monitoring of naturally occurring radioactive materials for regulation. Queensland University of Technology.

- Beretka, J. and Mathew, P. (1985): Natural radioactivity of Australian building materials, industrial wastes and by-products. Health Phys., 48(1): 87.

- Candeias, C.; Ávila, P.; Coelho, P. and Teixeira, J. P. (2018): Mining activities: health impacts. Reference Module in Earth Systems and Environmental Sciences, 1-21.

- Chandrasekaran, A.; Ravisankar, R.; Senthilkumar, G.; Thillaivelavan, K.; Dhinakaran, B.; Vijayagopal, P.; Venkatraman, B. (2014): Spatial distribution and lifetime cancer risk due to gamma radioactivity in Yelagiri Hills, Tamilnadu, India. Egypt. J. Basic Appl. Sci., 1(1): 38.

- De Lacerda, L.D. and Salomons, W. (2012): Mercury from gold and silver mining: a chemical time bomb? : Springer Science \& Business Media. 
- Doyi, I.; Oppon, O.; Glover, E.; Gbeddy, G. and Kokroko, W. (2013): Assessment of occupational radiation exposure in underground artisanal gold mines in Tongo, Upper East Region of Ghana. J. Environ. Radioact., 126: 77.

- Eisenbud, M. and Gesell, T.F. (1997): Environmental Radioactivity from Natural, Industrial \& Military Sources: From Natural, Industrial and Military Sources: Academic press.

- El Mamoney, M. and Khater, A.E. (2004): Environmental characterization and radio-ecological impacts of non-nuclear industries on the Red Sea coast. J. Environ. Radioact., 73(2): 151.

- Faanu, A.; Adukpo, O. K.; Kansaana, C.; TetteyLarbi, L.; Lawluvi, H.; Kpeglo, D. O.; Amoah, P. A. (2016): Impact assessment of naturally occurring radioactive materials on the public from gold mining and processing at Newmont Golden Ridge Limited, Akyem, Eastern Region of Ghana. Rad. Protec. \& Environ., 39(3): 155.

- Girigisu, S.; Ibeanu, I.; Adeyemo, D.; Onoja, R.; Bappah, I. and Okoh, S. (2014): Assessment of radiological levels in soils from artisanal gold mining exercises at Awwal, Kebbi state, Nigeria. Res. J. App. Sci. Eng. Technol., 7(14): 2899.

- Hilson, G. (2002): The environmental impact of small $\square$ scale gold mining in Ghana: identifying problems and possible solutions. Geographic. J., 168(1): 57.

- Hollaway, J. (1993): Review of technology for the successful development of small scale mining. Chamber of Mines J., 35(3): 19-25.

- Hu, B.; Wang, C.; Xu, X.; Zhang, S.; Bao, S. and Li, Y. (2016): Assessment of radioactive materials and heavy metals in the surface soil around uranium mining area of Tongliao, China. Ecotoxicol. Environ. Saf., 130: 185.

- ICRP. (2007): Recommendations of the International Commission on Radiological Protection ICRP. ICRP Publication 103: 37(2.4), 2.

- Jibiri, N.; Isinkaye, M. and Momoh, H. (2014):
Assessment of radiation exposure levels at Alaba ewaste dumpsite in comparison with municipal waste dumpsites in southwest Nigeria. J. Rad. Res. Appl. Sci., 7(4): 536.

- Kamunda, C.; Mathuthu, M. and Madhuku, M. (2016): An assessment of radiological hazards from gold mine tailings in the province of Gauteng in South Africa. Int. J. Env. Res. Pub. He., 13(1): 138.

- Khater, A.E.; Al-Mobarak, L.; Aly, A.A. and AlOmran, A. (2010): NORM in clay deposits. Paper presented at the Proceedings of Third European IRPA Congress.

- Kolo, M.; Baba-Kutigi, A. N; Olarinoye, I. and Sharifat, I. (2012): Assessment of natural radioactivity levels and radiation hazards in the tertiary institutions in Minna, Niger State, Nigeria. Continental $J$. Environ. Sci., 6(3): 25.

- Kolo, M.T.; Aziz, S.A.; Khandaker, M.U.; Asaduzzaman, K. and Amin, Y.M. (2015): Evaluation of radiological risks due to natural radioactivity around Lynas Advanced Material Plant environment, Kuantan, Pahang, Malaysia. Environ. Sci. Pollut. Res. Int., 22(17): 13127.

- Kolo, M.T.; Khandaker, M.U.; Amin, Y.M. and Abdullah, W.H.B. (2016): Quantification and radiological risk estimation due to the presence of natural radionuclides in Maiganga Coal, Nigeria. PloS One, 11: 6 .

- Kolo, M.T.; Khandaker, M.U. and Shuaibu, H.K. (2019): Natural radioactivity in soils around mega coal-fired cement factory in Nigeria and its implications on human health and environment. Arab. J. Geosci., 12(15): 481.

- Kuan, L.S.; Wagiran, H. and Ramli, A.T. (2009): Measurement of Natural Radioactivity in Soil Samples from Hotspot Areas Around Kampung Sungai Durian, Kinta District, Perak. Measurement, 7: 10.

- Meech, J. A.; Veiga, M.M. and Tromans, D. (1998): Reactivity of mercury from gold mining activities in darkwater ecosystems. Ambio, 92.

- Mireku-Gyimah, D. and Suglo, R. (1993): The state 
of gold mining in Ghana. Transactions-institution of mining and metallurgy, 102: a151.

- Morsy, Z.; El-Wahab, M.A. and El-Faramawy, N. (2012): Determination of natural radioactive elements in Abo Zaabal, Egypt by means of gamma spectroscopy. Ann. Nuc. Energy, 44: 8.

- Munyaradzi, Z.; Anna, K.N. and Makondelele, T.V. (2018): Excess lifetime cancer risk due to natural radioactivity in soils: Case of Karibib town in Namibia. African Rev. Phys., 13.

- Njinga, R.; Jonah, S. and Gomina, M. (2015): Preliminary investigation of naturally occurring radionuclides in some traditional medicinal plants used in Nigeria. J. Rad. Res. Appl. Sci., 8(2): 208.

- NYSDH. (2007): Radiation and Health. New York State Department of Health.

- Okore, R. (2018): Nigeria's thriving illegal gold mining activities and challenge of lead poisoning. Energy-TheGuardian Nigerian News.

- Osimobi, J.; Avwiri, G. and Agbalagba, E. (2018): Radiometric and Radiogenic Heat Evaluation of Natural Radioactivity in Soil Around Solid Minerals Mining Environment in South-Eastern Nigeria. Environ. Proc., 5(4), 859.

- Pure Earth. (2008): Artisanal Gold Mining.

- Sabo, A.; Sadiq, L.S. and Gamba, J. (2018): Radiological Assessment of Artisanal Gold Mining Sites in Luku, Niger State, Nigeria. J. Environ. Poll. Human Health, 6(2): 45.

- Sivakumar, S.; Chandrasekaran, A.; Ravisankar, R.; Ravikumar, S.; Jebakumar, J.P.P.; Vijayagopal, P.; Jose, M. (2014): Measurement of natural radioactivity and evaluation of radiation hazards in coastal sediments of east coast of Tamilnadu using statistical approach. J. Taibah Univ. Sci., 8(4): 375.

- Stranden, E. (1976): Some aspects on radioactivity of building materials. Phys. Norv., 8(3): 163.

- Suleiman, K.; Agu, M. and Onimisi, M. (2018): Evaluation of Naturally Occurring Radionuclide in Soil Samples from Erena Mining Sites in Niger State,
Nigeria. Curr. J. Appl. Sci. Technol., 1.

- Taskin, H.; Karavus, M.; Ay, P.; Topuzoglu, A.; Hidiroglu, S. and Karahan, G. (2009): Radionuclide concentrations in soil and lifetime cancer risk due to gamma radioactivity in Kirklareli, Turkey. $J$. Environ. Radioac., 100(1): 49.

- Turyahabwa, E.; Jurua, E.; Oriada, R.; Mugaiga, A. and Enjiku, B. (2016): Determination of natural radioactivity levels due to mine tailings from selected mines in Southwestern Uganda. Environ. Earth. Sci., 6(6): 154.

- UNSCEAR. (2000): Sources and Effects of Ionizing Radiation. Report to General Assembly, with Scientific Annexes. United Nations, New York.

- UNSCEAR. (2008): Effects of ionizing radiation: report to the General Assembly, with scientific annexes (Vol. 1): United Nations Publications.

- Vinay, K.R.K.; Gopal, R.C.; Vidya, S.D.; Yadagiri, R.P. and Rama, R.K. (2012): Environmental radioactivity studies in the proposed Lambapur and Peddagattu uranium mining areas of Andhra Pradesh, India. Rad. Prot. Dosim., 151(2): 290. 